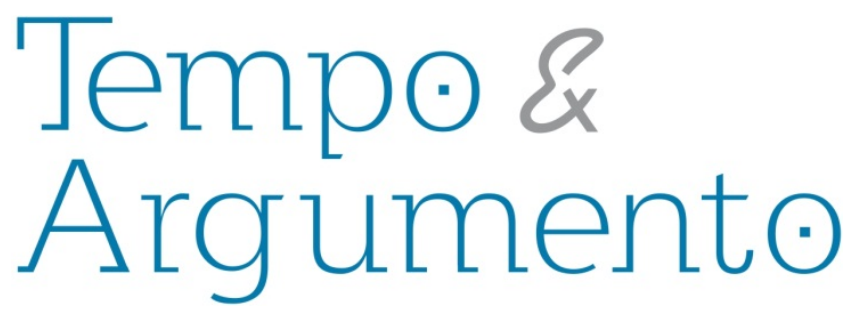

\title{
Entre mémoire et histoire, filiation saccagée, trauma, récit de vie et passion des archives
}

\begin{abstract}
Résumé
Mon intervention relate des aspects d'une recherche ou plutôt d'un compagnonnage avec un groupe particulier, d'anciens enfants juifs cachés en France pendant l'occupation qui ont survécu au génocide et que je rencontre 60 ans après. Mon propos va s'appuyer sur ma participation aux activités de l'Association pour la mémoire du convoi $Y^{1}$ qu'ils ont crée et dont je vais suivre les différentes activités puis réaliser des entretiens auprès de 16 participants. Pendant plus de quatre années, je vais suivre le groupe: les réunions, les assemblées générales, les voyages de commémoration en province, à Paris et en Pologne, les expositions et différents développements de l'association Pour la mémoire du Convoi $Y$ créée par le groupes. Le groupe ne s'est pas constitué au nom de leur expérience douloureuse er singulière d'enfants juifs cachés en France pendant l'Occupation, mais au nom de la mémoire, de leurs parents déportés en juillet 1942 et le plus souvent assassinés à Auschwitz. Je me suis demandé ce qui se jouait dans le dynamisme de ces démarches contemporaines sur des traumas et des disparitions de plus d'un demi-siècle, avec le sentiment que se remettait en route une possibilité créative d'élaboration des deuils et des perte comme autorisé ou légitimé par le projet de recherche, de transmission et une passion des archives.
\end{abstract}

Mots clefs: Enfants caches. Juifs. Déportation. Archives. Transmission. Deuxième guerre mondiale.

\author{
Yoram Mouchenik \\ Professeur de Psychologie \\ Clinique à l'Université Paris 13. \\ yoram.mouchenik@free.fr
}

\footnotetext{
Pour citer cet article :

MOUCHENIK, Yoram. Entre mémoire et histoire, filiation saccagée, trauma, récit de vie et passion des archives. Revista Tempo e Argumento, Florianópolis, v. 5, n.9, jan./jun. 2013. p. 218 $-230$.
}

DOI: $10.5965 / 2175180305092013218$

http://dx.doi.org/10.5965/2175180305092013218

\footnotetext{
${ }^{1}$ Nous garderons anonyme le nom de l'association et de ses participants par respect pour la confidentialité.
} 
Between memory and history, broken filiation, trauma, life story and passion for archives

\begin{abstract}
My paper describes some aspects of a research or rather a fellowship I conducted with a particular group of former Jewish children hidden in France during the occupation who survived the genocide and I met 60 years later. My purpose is based on my involvement with the Association for the memory convoy $Y$ they have created and I will follow the various activities and conduct interviews with 16 participants. For over four years, I will follow the group: meetings, general meetings, commemoration trips in province, in Paris and Poland, exhibitions and various developments of the association. The group did'nt met in the name of their singular and painful experience of Jewish children hidden in France during the WW II, but on behalf of the memory of their parents deported in July 1942 and mostly murdered in Auschwitz. I wondered what was at stake in the dynamism of those contemporary meeting about traumas and disappearances of more than half a century, with the feeling that recovering road and possible mourning were authorized or legitimized by their research project, transmission aims and passion for archives.
\end{abstract}

Keywords: Hidden Jewish. Children. Deportation. Archives. Transmission. World War II.

\section{Entre memória e história, filiação destruída, trauma, narrativa de vida e paixão por arquivos}

\section{Resumo}

Minha intervenção relata aspectos de uma pesquisa, ou antes, de um convívio com um grupo particular pessoas que foram crianças judias que permaneceram escondidas na Franca durante a Ocupação e que sobreviveram ao genocídio, e que eu encontrei 60 anos depois. Meu argumento vai apoiar-se na minha participação nas atividades da Associação pela memória do Comboio $\mathrm{Y}$, que elas criaram e cujas diferentes atividades eu segui, para depois realizar entrevistas com 16 participantes. Durante mais de quatro anos, acompanhei o grupo: as reuniões, as assembleias gerais, as viagens de comemoração no interior, em Paris e na Polônia, as exposições e as diferentes evoluções da associação criada pelo grupo. O grupo não se constituiu em razão de sua experiência dolorosa e singular de crianças judias escondidas na Franca durante a Ocupação, mas em nome da memória de seus pais deportados em julho de 1942, em sua maioria assassinados em Auschwitz. Perguntei-me o que estava em jogo no dinamismo dessas atitudes contemporâneas sobre traumas e desaparecimentos que datam mais de meio século, com o sentimento de que se reinstaurava uma possibilidade criativa de elaboração dos lutos e das perdas como autorizado ou legitimado pelo projeto de pesquisa sobre transmissão e uma paixão por arquivos.

Palavras-chave: Crianças escondidas. Judeus. Deportação. Arquivos. Transmissão. Segunda guerra mundial. 
Je vous propose une réflexion commune dont un domaine, les archives, dont je ne suis pas un spécialiste. Mon intervention relate des aspects d'une recherche ou plutôt d'un compagnonnage (MOUCHENIK, 2004, 2006) avec un groupe particulier, d'anciens enfants juifs cachés en France pendant l'occupation qui ont survécu au génocide et que je rencontre 60 ans après. Mon propos va s'appuyer sur ma participation aux activités de l'Association pour la mémoire du convoi $Y$ qu'ils ont crée et dont je vais suivre les différentes activités puis réaliser des entretiens auprès de 16 participants. Pendant plus de quatre années, je vais suivre le groupe : les réunions, les assemblées générales, les voyages de commémoration en province, à Paris et en Pologne, les expositions et différents développements de l'association Pour la mémoire du Convoi Y créée par le groupe.

\section{Le contexte}

L’immédiate après-guerre est marquée par l'irrecevabilité de la parole des juifs rescapés des camps d’anéantissement, appelés déportés raciaux, ils ne sont pas représentés lors des cérémonies du 11 novembre 1945, place de l'Étoile (GANDINI, 1999, p.98), Primo Levi (1947/1987) ne parvient pas à publier son témoignage, Si c'est un homme. Il faut attendre plusieurs dizaines d'années avant qu'un discours sur la collaboration, la déportation et l'anéantissement des juifs d'Europe trouve sa légitimité dans l'espace public. Ce silence rend certainement compte d'une forme de congélation de la mémoire jusqu'aux années 1980. En France, cette évolution récente est certainement balisée par les procès Barbie (1985), Touvier (1994), Papon (1997), le film Shoah de Jacques Lanzman en 1985, la reconnaissance par l'ancien Président de la République, en 1995 de la responsabilité de l'état français et de sa participation à la solution finale pour les Juifs de France. Cette reconnaissance est précédée par le travail considérable de serge Klarsfeld, non seulement avec Beate Klarsfeld comme militants anti-nazis, dont l'action sur l'opinion publique et le gouvernement Allemand oblige à juger d'anciens nazis, confortablement reconvertis (procès de Cologne, etc.), mais aussi par la production en 1978 du livre Mémorial des Juifs de France, basé sur une recherche archivistique en France, 
en Allemagne, en Pologne, etc., qui recense, l'ensemble de la déportation des juifs de France dont la police et la gendarmerie française ont procédé au recensement, aux rafles et aux arrestations. Serge Klarsfeld, dont le père est arrêté et déporté quand il avait cinq ans, entreprend de retrouver à travers les archives le nom de tous les déportés juifs de France, avec la date de leur déportation, le numéro du convoi de départ de France et la date et le lieu de leur assassinat quand ils sont connus. Ce livre, Le mémorial de la déportation des juifs de France (KLARSFELD, 1978) livre-sépulture, mais aussi archive d'archives est un véritable choc avec ces listes, ces noms, ces dates et numéro de convoi avec pour chaque convoi un bref historique, souvent la première épitaphe collective accessible à la famille. Cette publication et cette matérialisation dans l'espace public sont souvent, à l'époque, la première étape qui rend un travail de deuil possible ${ }^{2}$. La place du document et de l'archive est bien soulignée par Serge Klarsfeld pour lui-même : «Le Mémorial offrait par la lecture ce que j'avais éprouvé sur place à Auschwitz lorsque j'avais retrouvé le numéro de matricule de mon père dans le registre de l'infirmerie, suivi d'une croix. Ce jour-là ma quête s'est interrompue." (BOCHURBERG, 1997, p. 212)

Egalement fin des années 1980 c'est la réactivation des travaux sur les spoliations des juifs pendant l'occupation, les lois Juppé/Jospin sur les réparations, l’octroi de pensions, etc. Cet avènement de la possibilité d'une mémoire socialement reconnue va enclencher de multiples mouvements, associations, travaux, livres, et aussi enclencher cette demande archivistique et pour certains une passion des archives.

L'archive nous tourne vers le passé, mais je suis aussi de l'avis de Derrida qui voit l'archive comme un gage d'avenir. C'est à dire que l'archive qui permet de savoir autant que ce peut, est un itinéraire, une archive pouvant conduire à une autre, un fond d'archive à un autre, l'archive est donc le vecteur d'une temporalité et d'une continuité qui guide l'attention de Derrida : «L'archive a toujours été un gage, et comme tout gage un gage d'avenir » (DERRIDA, 1995, p. 37).

\footnotetext{
${ }^{2}$ Ce projet et sa réalisation a une portée considérable pour les familles et descendants des déportés juifs de France, symboliquement il donne une trace et inscrit à l'extérieur de soi, le nom des déportés en les figurant collectivement et par convoi successif, il génère la représentation groupale reprise par les descendants.
} 


\section{L'association du convoi Y et la demande d'archives}

Le groupe composé d'anciens enfants cachés ${ }^{3}$ en France, pendant l'occupation, ne s'est pas réuni en fonction de cette expérience commune, mais au nom de leurs parents déportés dans les camps d'anéantissement par un même convoi ferroviaire de plus de 900 personnes, que j'appellerai ici le Convoi Y, parti du Loiret pour Auschwitz en juillet 1942. C'est au nom de cette communauté de destin qu'ils vont se retrouver avec l'idée que la proximité de leurs parents déportés ensemble, et le plus souvent assassinés, leur confère un lien de parenté. Les premiers contacts, qui seront à l'origine de la création de la future association, vont se nouer lorsqu'un participant, Henri, met une annonce dans les journaux à la recherche de descendants de déportés des trois convois différents qui déportent pour les assassiner : son père, sa mère, son frère. II faudra encore trois années pour que le groupe s'étoffe et que par la volonté et la ténacité de quelques personnes, quatre-vingts participants se retrouvent chez Mme X pour la réunion fondatrice de l'association à laquelle je vais assister. Je me suis demandé ce qui se jouait dans le dynamisme de ces démarches contemporaines sur des traumas et des disparitions de plus d'un demi-siècle, avec le sentiment que se remettait en route une possibilité créative d'élaboration des deuils et de la perte comme autorisé ou légitimé par le projet de recherche dans les archives et de transmission.

II m’a rapidement semblé que se jouait là un itinéraire complexe où il est question de nombreux éléments problématiques dont on peut en énumérer certains :

- La filiation et la réinscription dans une chaîne généalogique,

- Un devoir de sépulture, injonction inévitable et paradoxale, sans corps, sans rites et sans sépulture,

- La question du deuil différé,

\footnotetext{
${ }^{3}$ Les enfants cachés et traqués en France doivent leur survie aux efforts désespérés de leurs parents ou des organisations de sauvetage des enfants juifs pour les mettre à l'abri. Ils sont le plus souvent confiés à des institutions religieuses chrétiennes ou à des familles d'accueil. Le terme "enfants cachés » ne devient un repère identitaire que récemment, permettant dorénavant à des centaines de milliers de personnes de par le monde de se reconnaître dans une expérience singulière. L'association des "Enfants cachés ", se crée notamment après la première rencontre internationale des enfants cachés qui se tient à New York en 1991.
} 
- Les processus auto-thérapeutiques pour réélaborer une enfance saccagée,

- Le maniement de la culpabilité liée à la survivance dans une situation de génocide,

- Les places de parents confrontés à une nécessité de transmission de l'histoire familiale et du traumatisme collectif historique dans des relations difficiles à leurs enfants.

- Un appétit insatiable de savoir sur les parents déportés qui va passer par la visite aux archives disponibles.

Dès la création de l'association pour la mémoire du convoi $Y$, la question de la recherche impérieuse d'archives comme la mise en commun d'archives familiales, etc., sont au cœur des projets de l'association. Dans cet appétit au-delà d'un devoir de piété filial et de travail de deuil, il s'est agi aussi à travers la recherche archivistique sous différentes formes sur le Convoi $Y$ de construire un support de connaissance et de transmission.

Freud a parlé d'une pulsion épistémophilique, pulsion de savoir, pulsion de connaissance à partir de la libido. Au-delà de Freud pour le psychanalyste René Kaës l'appétit d'archive est certainement inséparable de l'impérieuse nécessité de savoir pour transmettre : «(...) un phénomène qui pourrait être décrit comme une urgence ou comme une sorte de poussée à transmettre sous l'effet d'un impératif psychique incoercible (...) »(KAES, 1993, p. 8).

Car comme l'écrit Régine Waintrater (2003, p.19) : «Tout génocide est une catastrophe de la filiation : il est l'anéantissement simultané de trois générations, celles qui sont nécessaires à l'établissement de toute filiation, pour permettre que chacun puisse se situer dans un ensemble de sujets et se reconnaître comme ayant été engendré et capable d'engendrer ».

Il me semble qu'il faut voir la demande d'archive comme un processus d'historicisation

Je ne séparerai pas histoire et historicisation et comme l'écrit Annette Wievorka ; «L'histoire est aussi une des modalités du travail de deuil, tentant d'opérer - avec des difficultés de tous ordres -, l'indispensable séparation des vivants et des morts.» WIEVORKA (2005, p. 280). 
La démarche l'historicisation (ENRIQUEZ, 1988) et son besoin d'archives devient un besoin impérieux comme le souligne aussi François Maspéro dont le père, déporté politique, mourut à Buchenwald : «Étrangement, plus je me suis éloigné dans le temps, plus mon souci de voir clairement ce qu'a été la vie de mon père au camp s'est précisé ». (2002, p.29).

C'est une passion des archives qui saisit ces enfants de déportés participants à l'association du convoi Y. Gérard a un parcours complexe, qui évolue depuis des dizaines d'années autour de la déportation de son père revenu des camps et décédé depuis. Au début des années 1990, il participe pendant plusieurs années à un "groupe de paroles » d'enfants de survivants de la Shoah qui lui laisse une impression mitigée d'inachèvement, mais le relie enfin à une forme d’identité. Un déclic important intervient lorsque Gérard participe à une commémoration dans les camps d'internement du Loiret : « Je suis allé à la cérémonie de Pithiviers/Beaune-La-Rolande, tout à fait par hasard. Nous nous sommes retrouvés dans un petit groupe et l'idée que j'avais émis d'aller fouiller les archives les intéressait. C'est devenu une sorte de révélation. Nous sommes allés aux Archives Nationales en candide. La personne qui m'intéressait c'était mon père que j'ai connu et qui est mort. C'est au moment où l'on parlait beaucoup des spoliations, de la commission Drai et Mattéoli ${ }^{4}$. Je cherche mon père sur microfilm et il y a toute la famille de mon père qui apparaît et cela je ne m'y attendais pas du tout. Je fais pareil pour ma mère et il y a toute la famille de ma mère qui arrive et je ne m'y attendais pas du tout. Ce sont les fiches de Beaune-La-Rolande, de Drancy, de la Préfecture de Police, etc. Cela me marque sacrément car je ne connaissais pas l'histoire de mon père. Je savais vaguement qu'il était à Beaune-La-Rolande et déporté à Birkenau. Je sais que mes grands-parents ont été déportés aussi, mais dans quelles circonstances? C'est le passage de l'abstrait au concret. Tout d'un coup, tu as sous les yeux des éléments matériels qui décrivent la trajectoire de ta famille. »

L'accès et les recherche dans les archives marquent un tournant considérable dans

\footnotetext{
${ }^{4}$ Commissions d'indemnisation des spoliations économiques des Juifs pendant l'occupation, mises en place par les gouvernements Juppé et Jospin. D’une part la mission d'étude sur la spoliation des Juifs de France instituée en 1997, puis la commission d'indemnisation des victimes des spoliations du fait des législations antisémites pendant l'occupation.
} 
la représentation que Gérard se fait de sa famille et dans la dynamique personnelle et interpersonnelle qui s'enclenche : «Je me mets à fouiller vivement dans les archives et j’arrive à reconstituer, non pas un arbre généalogique, mais les relations entre différentes personnes. Il me reste très peu de famille. J'ai reconstitué une grande partie de la trajectoire de la famille de mon père à partir du moment où il est arrivé en France ainsi que pour la famille de ma mère. Il m'est arrivé un tas de choses à travers les archives, au travers des rencontres de gens qui avaient connu la famille de mon père avant-guerre. De L'absence d'histoire familiale, je me retrouve avec une histoire familiale douloureuse. »

La recherche de Gérard devient l'objet d'une véritable passion et une révélation qui donnent un sens à son parcours : «Dès que j’ai mis le nez là-dedans, je me suis senti dominé par cela. C'est quelque chose qui me domine, c'est ce que je dois faire. Cela n'est pas uniquement pour moi, il se trouve que j'ai des enfants en bas-âge et cela me paraît être en relation avec eux. Quand je regarde mon parcours, je m'aperçois que toute ma vie a tourné autour de ce qui s'est passé pendant la seconde guerre mondiale, les camps, sans que je m’en aperçoive. Je suis né en 1948. Mon parcours a été déterminé par les camps. Mon père ne parlait qu'en termes de camps. Rien n'était dit directement par mon père, ce que j'ai pu savoir, c'est quand il s'adressait à un tiers. (...) En tant qu'adulte, c'était aussi impossible d'en parler avec mon père, de son fait et du mien. Toutes les questions que je me pose, il aurait pu, il y a dix ans m'en parler, j'aurai pu lui poser les questions, il m'aurait répondu. Il se trouve que cela ne s'est pas fait (FRESCO, 1981) ${ }^{5}$. Je ne culpabilise pas là-dessus. Je suis enfant unique. Je n'ai pas eu une relation simple, mon père n'était pas un mec souple, ni très à l'écoute. Il était capable de se bloquer sans explication. » Ou bien Martine : «Résumer la vie de mon père en quelques lignes car je ne sais rien d'autres de lui, c'est très douloureux, mais une fois que c'était fait, je me suis dit, il existera pour les descendants. On a très peu d'héritage, une photo floue, presque rien. Dans cette recherche, j'ai retrouvé des traces dans les archives. Mon père a fait le

\footnotetext{
${ }^{5}$ Nadine Fresco (1981) décrit l'emprise du silence ou les révélations assourdissantes adressées à la première génération née après la Shoah: L'auteur nous dit: «(...) depuis l'enfance, le silence avait été trop absolu pour que le faire-part fût totalement une surprise. » (1981: 206) Pour d'autres, c'est un mutisme total sur un monde de douleurs et de souffrances cachées et des « drames interdits d'accès. » (Idem : 208), mais dont les indices multiples soulignent l'ampleur des deuils impossibles. L'auteur nous dit qu'à ces adolescents-là étaient transmise la blessure, mais pas la mémoire.
} 
maximum, il s'est engagé. »

Il faut aussi penser la demande archivistique comme celle qui s'adresse aux Lieux de mémoire et notamment les voyage à Auschwitz comme dans le cheminement d'Ernest ; devenu historien après sa retraite. Adolescent Ernest tombe malade en juin 1942 et part en sanatorium, ce qui lui permet d'échapper à la rafle du Vel-d'hiv de juillet 42. II nous dit :"Quand je me suis marié, les premières années, dans mon sommeil, je commençais à crier, je cherchais mon père et il n'était pas là, ou j'étais fâché avec lui, dans des cauchemars terribles où je me mettais à hurler, ma femme me calmait. Ce n'est qu'en 1981, pour le $39^{\mathrm{e}}$ anniversaire de la déportation, que le Rabin Farhi avec son association des Juifs libéraux a décidé de faire un pèlerinage à Auschwitz. J’y suis allé avec ma femme et c'est à ce moment-là que j'ai fait mon deuil. Quand je suis revenu, je n'ai plus eu de cauchemars, c'était fini. Le cheminement de ma pensée s'est fait du jour au lendemain, quand j'ai retrouvé le lieu, je connaissais les conditions, j'avais retrouvé à plus de 50 ans, mon équilibre par rapport à mon père. J'en ai fait le deuil, c'était fini, et ensuite toute ma vie, je me suis intéressé au judaïsme. » Avec une thèse d'histoire et plusieurs ouvrages qui font autorité sur le judaïsme d'Europe de l'Est avant-guerre.

Il faut comprendre la demande archivistique comme un phénomène complexe avec une conjonction de différents aspects : une nouvelle reconnaissance de la Shoah et de la collaboration en France à travers des lois et pensions réparatrices mais où il faut apporter des preuves sur les spoliations et donc utiliser les fond d'archive. La constitution de nouvelles associations à partir de la reconnaissance de l'existence de nouveaux groupes comme celui des enfants cachés ; première réunion internationale en 1991 à NewYork qui va générer de multiple association de par le monde et des démarches plus individuelles.

\section{Quelle compréhension de ces phénomènes}

Je proposerai quelques pistes de réflexion et de transversalité entre nos différentes disciplines. Nous avons trouvé, entre autre, comme métaphore pour comprendre ces phénomènes d'une part les processus de réinscription comme une forme 
de refus de faire de la Shoah l'unique événement fondateur ancestralisé comme point de départ de la lignée. Ceci va dans le sens du concept de « réinscription » et de transmission d'une mémoire du vécu ante-génocidaire, développé d'un point de vue anthropologique par Lemée et Galay (2003).

Le génocide n'était pas seulement une extermination mais une tentative d'effacement, «ils n'auront pas été ». Aussi cet aspect devenant quelques fois compulsifs de retrouver des archives est à la hauteur de cet enjeu, combattre une tentative de déexistence, la négation ce qui aura été, et ce qui peut advenir : la descendance et la culture. Nous évoquons les hypothèses complexes mais fructueuses de Bergès sur la « négation du futur antérieur » à propos du deuil et de la dépression chez l'enfant (1996) que l'on peut éventuellement élargir à notre propos. Ce n'est pas « il n'est plus », mais «il n'aura pas été ».

Nous pouvons revenir à Derrida et à sa critique de I'historien anglais Joseph Yerulshalmi n'acceptant pas le scénario de Freud dans son Moïse (1939), la thèse de Freud à l'image de Totem et Tabou (1912) est que Moïse l'Egyptien est assassiné par les Hébreu au seuil de la terre promise et que ce meurtre à l'instar de celui du père de la horde est refoulé (totalement oublié ou forclos), Derrida rappelle la place centrale du refoulement dans l'œuvre freudienne et la remarque de Yerushalmi (1984), si ce meurtre avait eu lieu, la Thora (bible hébraïque) qui est prolixe à ces sujets en aurait parlé. Derrida revient sur le refoulement comme mode d'archivage : "Simplement, ajoute-t-il, les textes de cette archive ne sont pas lisibles par "I'histoire ordinaire" et c'est tout l'intérêt de la psychanalyse, si elle en a un.»

Ce commentaire est important aussi sur une autre direction qui est la transmission transgénérationnelle du traumatisme. Comme si la demande impérieuse d'archives était aussi là pour donner un sens ou faire sortir du refoulement cette transmission inconsciente du traumatisme de la génération précédente. Ainsi le concept de postmemory développer aux USA par Marianne Hirsch peut être utile. Marianne Hirsh propose la notion de « post-mémoire » pour désigner cet écho indirect d'un événement traumatique, qui n'est pas médiatisé par des souvenirs mais par un investissement imaginaire et une initiative créatrice (HIRSH, 1997). C'est ainsi comme la construction 
d'une mémoire quasi personnelle sur des évènements que l'on n'a pas vécu, c'est-à-dire la prise en charge par les descendants d'un passé traumatique dont ils héritent personnellement. C'est un phénomène que Marianne Hirsch va particulièrement étudier à partir du livre Maus de Art Spiegelman (1986) sur l'expérience de la Shoah de son père déporté survivant ; Vladek. Ce livre sous forme de bande dessiné, n'est pas un exercice de style, il semble s'imposer à Spiegelman à partir de sa relation très difficile avec ce père revenu de déportation; mais aussi l'omniprésence de son frère mort pendant la guerre et le suicide de sa mère. Les enfants et petits-enfants de déportés investissent les récits de leurs parents pour alimenter leurs propres mémoires et s'inscrire dans une trajectoire collective. Les recherches dans les archives sont un des piliers de cette construction de la mémoire.

\section{Conclusions}

Il nous semble qu'il faut comprendre la demande d'archives comme un phénomène complexe qui ne peut s'appréhender que dans la transversalité de différentes disciplines et la psychologie à partir d'un travail de terrain peut apporter une modeste contribution.

\section{Références}

BERGES, Jean. Les états dépressifs de l'enfant. Conférence de l'hôpital Sainte Anne (conférence dactylographiée non publiée, 1996).

BOCHURBERG, Claude. Entretien avec Serge Klarsfeld. Paris: Stock, 1997.

DERRIDA, Jacques. Conférence prononcée le 5 juin 1994 à Londres lors d'un colloque international : Memory: The Question of Archives. Le texte est daté du 22-28 mai 1994.

ENRIQUEZ, Micheline. L'enveloppe de mémoire et ses trous. Topique 42, p.185-205, 1988. 
FREUD, Sigmund. Totem et tabou. Paris: Payot, 1968.

. Moise et le monotheisme. Gallimard: Paris, 1939/1967.

FRESCO, Nadine. La diaspora des cendres, Nouvelle Revue de Psychanalyse, Paris, l’Emprise, 24, p. 205-220, 1981.

GANDINI, Jean-Jacques. Le procès Papon. Paris: Librio, 1999.

HIRSH, Marianne. The Generation of Postmemory, Poetics Today, Durham, 29, 1, p. 103128, 2008.

. Family Pictures: Maus, Mourning and Post-Memory, Discourse, 15, 2, p. 3-29, 1992.

KAES, René.Transmission de la vie psychique entre generations. Paris: Dunod, 1993.

KLARSFELD, Serge. Le Mémorial de la déportation des Juifs de France. Paris: FFDJDF, 1978.

LEMEE, Carole.; GALAY, Daniel. L'Après-Shoah: des traumas aux processus de réinscription, Face à Face, 5, 2003.

MASPERO, François. Les abeilles et la guêpe. Paris: Seuil, 2002.

LEVI, Primo. Si c'est un home. Paris: Julliard, 1987.

MOUCHENIK, Yoram. De la disparition au deuil chez les orphelins de la Shoah, L'autre, 5, 1, p.129-141, 2004.

. Ce n'est qu'un nom sur une liste, mais c'est mon cimetière, Grenoble: La Pensée Sauvage, 2006.

SPIEGELMAN, Art. Maus : Un survivant raconte. 1986

WAINTRATER, R. Sortir du génocide. Témoignage et survivance. Paris: Petite bibliothèque Payot, 2011.

WIEVORKA, Annette. Auschwitz, 60 ans après, Paris: Robert Laffont, 2005.

YERUSHALMI, Yosef.; HAYIM, Zakhor. Histoire juive et mémoire juive. Paris: La découverte, 1984. 
